\title{
Giving preparative thin layer chromatography some tender loving care.
}

John J. Hayward*, Lavleen Mader \& John F. Trant*

${ }^{1}$ Department of Chemistry and Biochemistry, University of Windsor, Windsor, Ontario, Canada

jhayward@uwindsor.ca; j.trant@uwindsor.ca

\begin{abstract}
:
Preparative thin layer chromatography (prepTLC) is a commonly used method of purification suitable for small scale reactions. However, descriptions of the preferred methodology to load, run, and recover samples from prepTLC are non-standard and varied, making it part of the "hidden curriculum" of laboratory technique. In this article we report on the simple, costeffective methods we use to load and collect samples from a plate, which enhance the convenience, speed, and precision of this technique.
\end{abstract}

Keywords: Laboratory technique, chromatography, separation science, thin layer chromatography, tools

\section{Summary Scheme:}

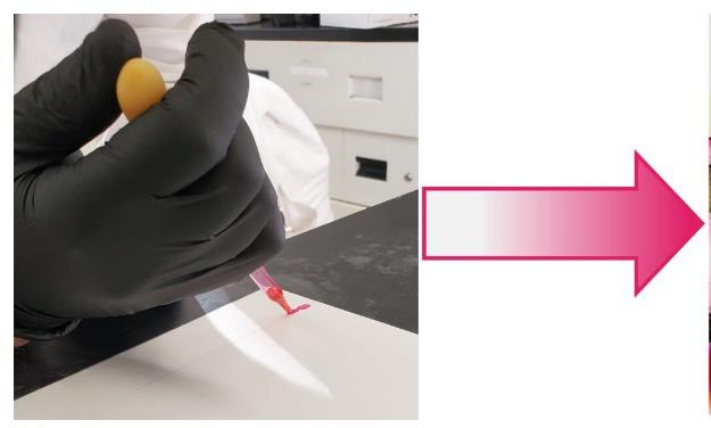

Application

Development

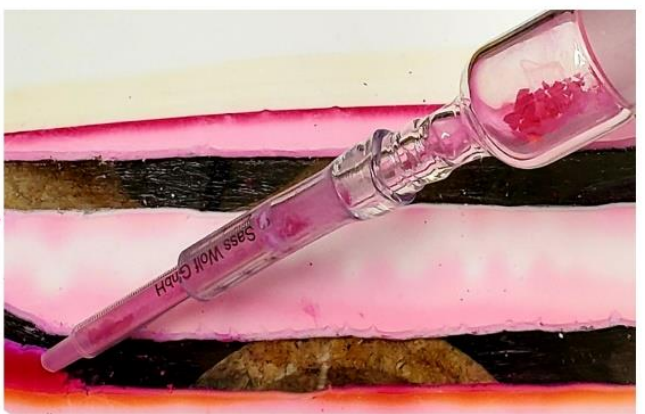

Extraction 
Introduction: Preparative thin layer chromatography (prepTLC) is a useful technique for small scale purification (typically $<100 \mathrm{mg}$ ) of compounds with very similar retention factors $\left(\mathrm{R}_{\mathrm{f}}\right)$ that co-elute through flash column chromatography. We are defining the technique as the use of rectangular or square, usually glass, plates coated with a layer $(250-2000 \mu \mathrm{m}$ thick $)$ of stationary phase; this is usually compacted ultra-fine silica, but alumina or C-18 functionalized silica are also available. Leonard, Lygo and Procter note that "prepTLC should definitely be considered a thing of the past,"1 and that it offers no benefits over preparative HPLC; however, as a lab that uses both extensively, we politely disagree - we have found it a useful and effective technique in several recent and ongoing projects. ${ }^{2}$ The ability to conduct multiple serial developments while observing in real time the relative change in separation of the components of interest is a significant advantage of this technique, as is the lack of a need to develop a specific gradient protocol, or the need to conduct initial purifications to remove components that would compromise an HPLC column. Furthermore, prepTLC generally requires little formal training. It is possible that this apparent simplicity and perceived obsolescence is the reason that many of our desktop references for experimental organic chemistry provide only a cursory discussion of the technique. ${ }^{3}$ This view is not universal of course, there are several excellent resources available in print and online discussing the theory and practice of the technique ${ }^{4}$ however, technical challenges in applying and recovering samples from the plates remain. Poor application of the sample to the plate can result in scratched silica and wide loading bands, both of which inherently compromise resolution; furthermore, incomplete removal of the silica and poor pre-treatment can lead to low percent recoveries and impure samples. We were inspired to write this article as it has become clear to us (JJH, JFT) that these two issues are the basis of many of the challenges faced by others when using prepTLC, and we wish to discuss some practices we employ to minimize these challenges and make prepTLC an incredibly powerful and versatile tool in our lab. 


\section{TLC sample applicator}

A vital component of a successful prepTLC procedure is the careful application of the crude mixture to the plate in a manner that avoids broadening of the baseline and ensures uniform sample loading A variety of methods have been reported, including the use of cotton swabs, ${ }^{5}$ rubber pipette bulbs, ${ }^{6}$ arrays of micropipettes, ${ }^{7}$ plastic $\operatorname{cups}^{8}$ and even strips of staples. ${ }^{9}$ For additional precision, designs requiring some engineering also exist, ${ }^{10}$ as do those requiring some glassblowing experience. ${ }^{11}$ Most of these describe themselves as simple and/or inexpensive, although not all. ${ }^{12}$ In addition, effective commercial applicators exist; however, these are expensive - prohibitively so for most laboratory settings, and occupy a large footprint. We have found that an easy method for sample application to a plate involves creation of a cotton-tipped "pen" from cotton wool and a glass pipette. This is made by taking a small amount of cotton wool (avoid the use of glass wool) and compacting it into the neck of one pipette; sufficient pressure for compaction can typically be applied by using a second, clean pipette (Figure 1a.). The pipette is then scratched about one third of the way along the cotton wool using a commercial glass cutting stone (in this case bought from Home Depot) and snapping along the scratch, to give the fabric tip of the pen (Figure 1b. \& c.). A pipette bulb can then be used to load the pen (Figure 1d.); the full volume of solution is not drawn up in one go: some slack should remain in bulb, and the cotton is aspirated briefly in air to dry the tip of the pen out somewhat. The pen can then be easily used to paint the compound onto the TLC plate along a pencil line (Figure 1e. \& f.). The presence of the cotton wool gives better control of the rate at which the solution is dispensed from the pipette, which allows for smooth and uniform deposition, while also not scratching the silica (Supplementary Video S1 \& S2).

Note: with especially difficult separations care must be taken to ensure the TLC plate has sufficient time to dry between applications to prevent excessive spreading of the solution: diffusion of the band decreases the resolution of the separation. The lower down the pipette the 
cotton can be pushed, and the lower the cut, the thinner the pen line. A flow of nitrogen is effective for drying the application line between passes with the pen. Should one be loading a sample in a high boiling point solvent, such as DMSO or water, gentle heating with a heat gun between passes is also effective. Incomplete drying leads to widening of the band, and a concomitant decrease in the expected resolution of the separation.

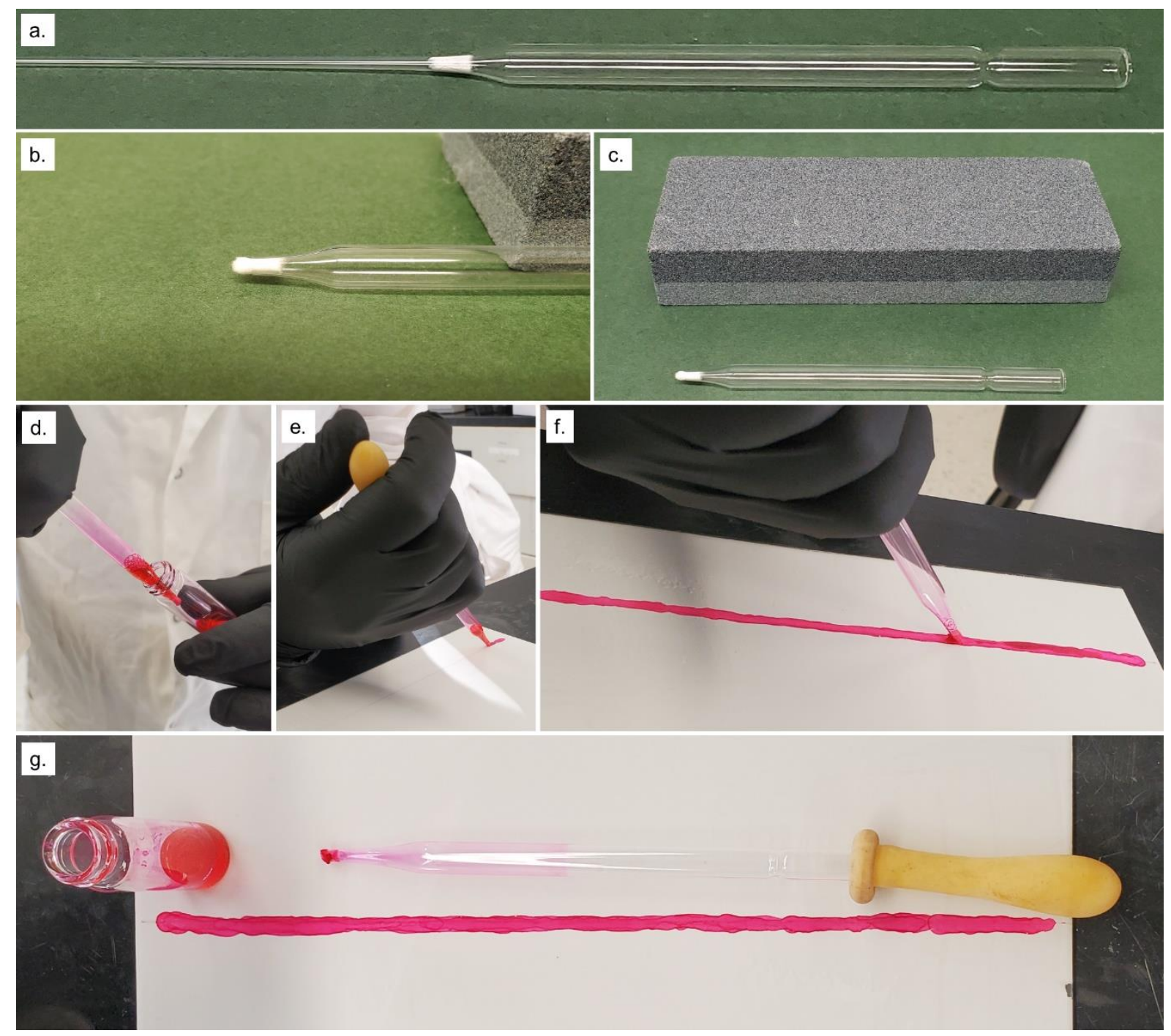

Figure 1 Construction and use of the TLC applicator pen. Applicator and vial included for scale.

\section{TLC zone extractor}

In contrast to the wide variety of methods available for application of compounds, methods for the easy collection of purified materials from the plate are comparatively scarce. Most sources 
instruct the practitioner to scrape the silica from the plate using a scalpel or spatula; however, this method often results in loss of silica to the surroundings, consequently lowering isolated yield. Devices involving a vacuum have been reported: a simple pipette with a cotton filter has been used, ${ }^{13}$ although this method has been criticised for the small volumes it is able to effectively extract; ${ }^{14}$ another solution involves several pieces of glassware and is ultimately quite bulky. ${ }^{15}$

We have found that a simple solution to this problem can be constructed from two gas inlet adapters. The first one has an outer joint, extended and narrowed by a broken off $1 \mathrm{~mL}$ syringe tip that is attached using a small length of PVC tubing (Nalgene $1 / 4^{\prime \prime}$ ID $\times 3 / 8$ " OD, Figure 2a.); the second adapter has a glass frit and a matching inner joint (Figure 2b.) that can then be attached to the house vacuum, water aspirator or other vacuum source. Connecting the two adapters together creates a device that can be used to collect silica directly from the plate with minimal loss of material; rubbing the syringe tip firmly onto the silica causes it to loosen and be sucked directly into the apparatus (Supplementary Video S3 \& S4), where it is collected against the frit; once some of the silica has been cleared from the glass backing, rubbing the apparatus along the side of the silica band is an effective and controlled method of uptake (Figure 2c.; Video S5). The band of interest can also be first scored with a sharp object such as a scalpel or syringe needle, and loose silica can likewise be collected (Video S6). For particularly narrow bands, a large gauge needle can be fixed to the syringe tip for additional precision (Figure 2 d.). Gentle tapping of the apparatus against the side of the bench while turned upright results in the silica collecting against the frit (Figure 2e.). The contents of the adapter can then be transferred to a filter for compound recovery. We have investigated the use of inlet adapters with B19/22, B24/40 and B29/42 joints; we found that extractors constructed from the B19/22 and B24/40 adapters were preferable, as the size of the B29/42 joints could 
result in a loss of dexterity. Furthermore, even for relatively wide compound bands the internal collection volume of the B19 or B24 extractor was sufficient.
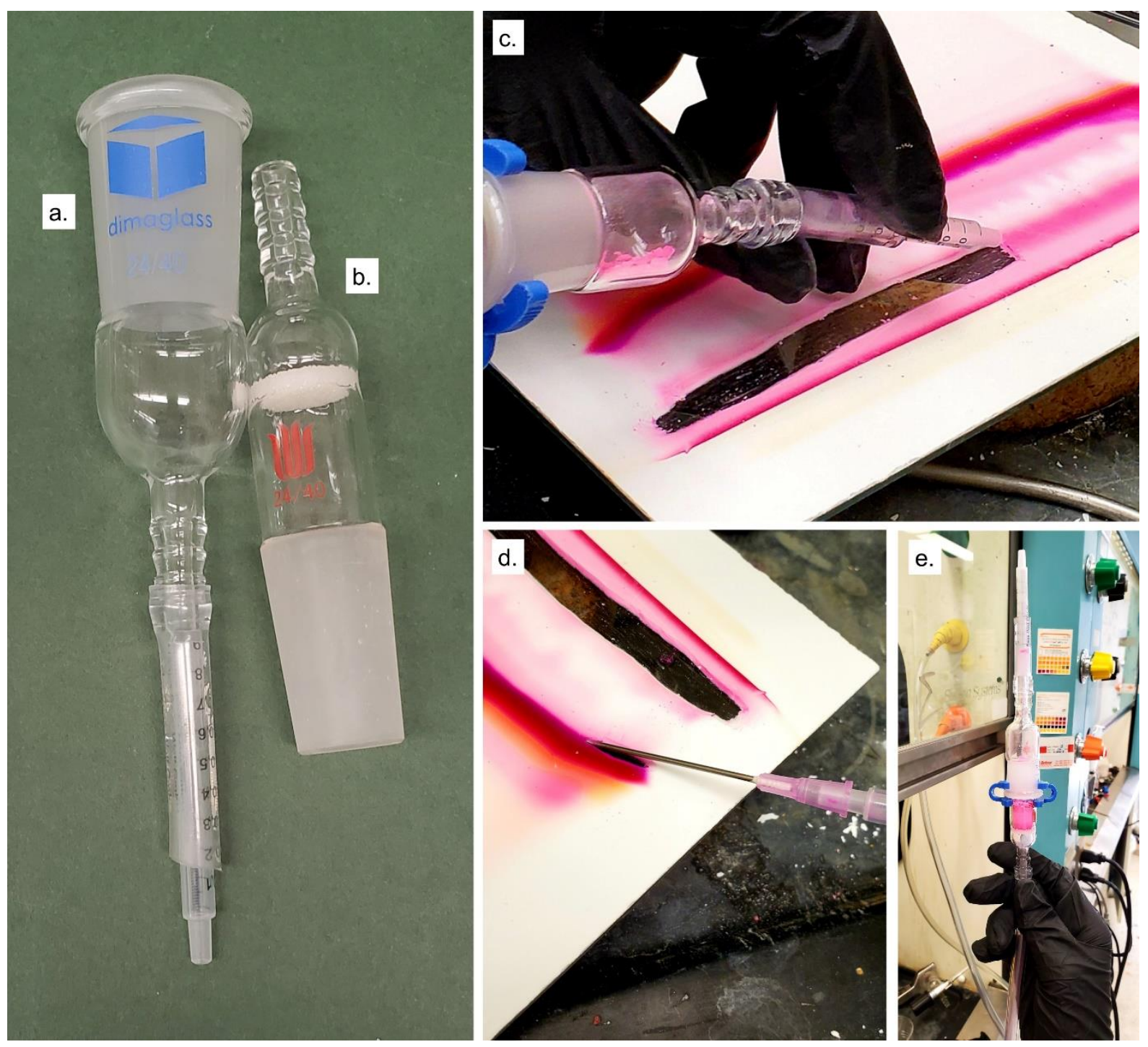

Figure 2 TLC zone extractor construction and use.

\section{Sample elution from silica}

One of the significant challenges sometimes encountered in prepTLC is the presence of hydrophobic "grease" impurities. This can, at times, feel ubiquitous in all spots of the plate, especially when isolating very small quantities of material. To avoid this, in the case of all samples that are not highly non-polar we load the silica from our extractor onto a fritted funnel, compact the silica by applying vacuum, and pass 3-10 column volumes of distilled pentane 
through the column. This greatly reduces the amount of hydrophobic impurities present in the isolated product after the elution.

There are various methods used to elute the compound from the silica following zone extraction. These largely fall into either stirring the silica in a relatively polar mixture and then filtering it off to extract the compound, or loading the silica directly onto a frit and eluting the compound using a polar solvent, essentially treating it as a miniature silica plug. The choice of solvent must ideally be far more polar than the compound, but still be capable of solvating the compound. We suggest a default of either distilled dichloromethane/methanol mixtures, or $100 \%$ distilled ethyl acetate followed by mixtures of methanol in ethyl acetate depending on solubility. A 1:1:1:X solution of water:methanol:acetonitrile:ethyl acetate is effective for particularly polar compounds. In this last ratio the volume of ethyl acetate can be varied to decrease or increase the polarity of the system, but the other three components should be kept in an equivolume ratio to maintain miscibility and appropriate viscosity. Ideally, all solvents used for extraction should be highly pure; we recommend using either HPLC grade solvent, or solvents predistilled by the operator.

Note: this 1:1:1:X solvent mixture is also effective as a mobile phase for extremely polar compounds.

\section{Conclusion}

Challenges in prepTLC often arise from improper application or extraction of the sample from the plate. These simple, convenient, and inexpensive methods for the application and extraction of compounds from a prepTLC plate resolve these problems. Of particular note is the zone extractor, which can be constructed very easily and substantially reduces the difficulty of this procedure. 


\section{Experimental Section:}

Solvents were purchased from Caledon Labs (Caledon, Ontario), Sigma-Aldrich (Oakville, Ontario) or VWR Canada (Mississauga, Ontario). Other chemicals were purchased from Sigma-Aldrich, AK Scientific, Oakwood Chemicals, Alfa Aesar or Combi-blocks and were used without further purification unless otherwise noted. Preparative TLC was done using glass-backed silica plates (Silicycle or Sanpont) of either 250, 500, 1000 or $2000 \mu \mathrm{m}$ thickness depending on application.

Glass pipettes were purchased from VWR, cotton wool from Dollarama and the glass cutting stone from Home Depot. Luer lock, $1 \mathrm{~mL}$ Injekt ${ }^{\circledR}$ syringes from B. Braun were used, PVC tubing was purchased from VWR or Thermo Scientific, and gas inlet adapters were purchased from Dimaglass and Synthware Glass (Kemtech America). The sintered glass frit within the inlet adapter was extra coarse.

Silica extracted from the plate was added to a fritted funnel, washed with pentane, and then the compound was eluted with an appropriate solvent, which was subsequently removed under reduced pressure on a rotary evaporator (Büchi).

\section{Acknowledgements}

The authors declare no competing financial interest. This study was supported by the Natural Sciences and Engineering Research Council of Canada \#2018-06338 (JFT) and the Government of Ontario \#ER18-14-114 (JFT-salary for JJH).

\section{Authors' Contributions}

Conceptualization: JJH and JFT; Funding acquisition: JFT; Investigation: JJH, LM, JFT; Methodology: JJH and JFT; Project Administration: JFT; Supervision: JJH and JFT; 
Visualisation: LM and JJH; Writing Original Draft: JJH and LM; Writing - review and editing:

All authors.

\section{References}

1. Leonard, J.; Lygo, B.; Procter, G., Advanced Practical Organic Chemistry. 3rd ed.; CRC Press: Boca Raton, FL, 2013.

2. Reynolds, M. R.; Piazza, S.; Chiaramonte, J.; Chapa-Villarreal, F. A.; Trant, J. F. New J. Chem. 2021; Bakht, M. K.; Lovnicki, J. M.; Tubman, J.; Stringer, K. F.; Chiaramonte, J.; Reynolds, M. R.; Derecichei, I.; Ferraiuolo, R.-M.; Fifield, B.-A.; Lubanska, D.; Oh, S. W.; Cheon, G. J.; Kwak, C.; Jeong, C. W.; Kang, K. W.; Trant, J. F.; Morrissey, C.; Coleman, I.; Wang, Y.; Ahmadzadehfar, H.; Dong, X.; Porter, L. A. J. Nuc. Med. 2020, 61, (6), 904-910; Bakht, M. K.; Hayward, J. J.; Shahbazi-Raz, F.; Skubal, M.; Tamura, R.; Stringer, K. F.; Meister, D.; Venkadakrishnan, V. B.; Xue, H.; Pillon, A.; Stover, M.; Tronchin, A.; Fifield, B. A.; Mader, L.; Ku, S.-Y.; Cheon, G. J.; Kang, K. W.; Wang, Y.; Dong, X.; Beltran, H.; Grimm, J.; Porter, L. A.; Trant, J. F. Proc. Natl. Acad. Sci. U.S.A. 2021, In Press; Taimoory, S. M.; Sadraei, S. I.; Fayoumi, R.; Nasri, S.; Revington, M.; Trant, J. F. J. Org. Chem. 2018, 83, 4427-4440; Sadraei, S. I.; Yousif, G.; Taimoory, S. M.; Igbokwe, E.; Mehri, S.; Alolabi, R.; Toma, J.; Rahim, M.; Trant, J. F. Preprint on ChemRxiv 2021.

3. Mohrig, J. R.; Hammond, C. N.; Schatz, P. F., Techniques in Organic Chemistry. 3rd edition ed.; W. H. Freeman and Company: New York, 2010; Pirrung, M., Handbook of Synthetic Organic Chemistry. 2nd ed.; Elsevier: Amsterdam, 2017; Cranwell, P. B.; Harwood, L. M.; Moody, C. J., Experimental Organic Chemistry. 3rd ed.; Wiley: Hoboken, NJ, 2017; Isac-García, J.; Dobado, J. A.; Calvo-Flores, F. G.; Martínez-García, H., Experimental Organic Chemistry. 1st ed.; Elsevier: Amsterdam, 2016; Sharp, J. T.; Gosney, I.; Rowley, A. G., Practical Organic Chemistry. 1st ed.; Chapman and Hall: London, UK, 1989; Caron, S., Practical Synthetic Organic Chemistry. 2nd ed.; Wiley: Hoboken, NJ, 2020. 4. $\quad$ Brooks, J. L.; Spencer III, W. T. How to Run Prep TLC. http://www.chem.rochester.edu/notvoodoo/pages/how_to.php?page=run_prep_tlc (12-112021), ; Nyiredy, S., Preparative Layer Chromatography. In Handbook of Thin-Layer Chromatography, 3rd ed.; Sherma, J.; Fried, B., Eds. Marcel Dekkar Inc: New York, 2003; Vol. 89, pp 399-441.

5. $\quad$ Sharma, R. J. Chem. Ed. 1991, 68, (1), 70.

6. Lo, J. M. J. Chem. Ed. 1982, 59, (1), 66.

7. Walsh, J. M. J. Chem. Ed. 1971, 48, (6), 415.

8. $\quad$ Lavallee, P.; Muzart, J. J. Chem. Ed. 1982, 59, (3), 250.

9. $\quad$ Price, H. C. J. Chem. Ed. 1974, 51, (4), 267.

10. Alhadeff, E. S. J. Chem. Ed. 1969, 46, (4), 249; Altman, L. J.; Trudell, J. R. J. Chem. Ed. 1970, 47, (5), 404; Malik, N. S. A.; Berrie, A. M. M. J. Chem. Ed. 1979, 56, (10), 680;

Fisher, T. L.; Gilman, C. P. J. Chem. Ed. 2001, 78, (3), 367.

11. Kim, Y. J. J. Chem. Ed. 1998, 75, (5), 640.

12. Fisher, T. L. J. Chem. Ed. 1983, 60, (11), 989.

13. Ruchelman, M. W. J. Chem. Ed. 1967, 44, (2), 110.

14. Kan, G.; Snieckus, V. J. Chem. Ed. 1969, 46, (11), 763.

15. Hansbury, E.; Ott, D. G. J. Chem. Ed. 1970, 47, (3), 229. 